\title{
Suicidal Risk Assessment in Hospitalized Bipolar Patients in Al-Mamoura Mental Health Hospital
}

H.E.El-Shiekh ${ }^{1}$, H.M.El-Sayed ${ }^{1}$, S.T.El-Bakry ${ }^{1}$ and I.M.Ibrahim ${ }^{2}$

${ }^{1}$ Neuropsychiatry Dept., Benha Univ., Benha, Egypt

${ }^{2}$ Al-Mamoura Mental Health Hospital, Alexandria, Egypt.

E-mail: Drultras7@gmail.com

\begin{abstract}
Suicide may be an endpoint for many patients with severe psychiatric illness. The mood disorders (depression and bipolar manic-depression) are by far the most common psychiatric conditions related to suicide. At least $25 \%$ to $50 \%$ of patients with bipolar disorder also attempt suicide at least once. The aim of the study was to evaluate the suicidal intent in bipolar hospitalized patients. In this descriptive cross sectional study, the number of patients who participated in the research and finished all the psychometrics were 113 patients, 73 males and 40 females. Psychiatric sheet of General Secretariat of Mental Health, Structured interview using Diagnostic and Statistical Manual of Mental Disorders, Fourth Edition (DSM IV) and Arabic version of Beck Scale for Suicide Ideation (BSS) were done for all patients. According to beck scale, $51.3 \%$ of the studied patients had no suicidal idea, $26.3 \%$ had passive suicidal ideation, $16.8 \%$ had active suicidal ideation and 5.3\% had active and passive ideas at the time of study. It was concluded that suicidal risk is high among bipolar disorder patients, also unemployment, family history of suicide and longer duration of illness are considered a risk factor for suicidal attempts and ideation.
\end{abstract}

Keywords: Suicide, Bipolar, Beck scale, Risk factors, Suicidal ideation.

\section{Introduction}

Bipolar disorder is a mental disorder that causes episodes of depression and episodes of elevated mood. The elevated mood is significant and is known as mania or hypomania depending on severity or presence of psychotic symptoms [11].

Mood disorders are the most common chronic psychiatric disorders in the world and are a leading cause of morbidity. In patients with these disorders mood can range from elation or mania to deep depression. Patients with bipolar I disorder typically show at least one major manic episode and usually also a major depressive episode while patients with bipolar II disorder typically demonstrate a pattern of depressive symptoms and hypomanic episodes [3].

Suicide is the deliberate act of ending one's own life [4]. People who attempt suicide may have a true desire to die, or this behavior may just be a response to pain, despair and hopelessness-a cry for help [2].

According to the World Population Review rankings in 2018, Egypt ranked in the bottom 10 countries globally for the number of suicides per capita, with 2.6 per 100,000 people. The World Health Organization (WHO) reported that the suicide mortality rate in Egypt was four cases per 100,000 people in 2016. But anecdotal evidence suggests the rate is higher than statistics show - or at the very least, cases of suicide have become more prominent in traditional and social media.

Suicide is a complex behavioral endpoint resulting from a group of factors. One of the most important of these is mental illness. People with bipolar disorder are among the highest rate of suicide compared to those with other mental disorders [14]. Bipolar disorder is associated with an estimated rate of suicide deaths of 0.2 to 0.4 per 100 person-years [7] and a standardized mortality ratio of 10-30 times that of the general population [13].

A study comparing 482 bipolar disorder suicides in Taiwan to suicide deaths without a recorded psychiatric diagnosis [20] found that bipolar disorder suicides were significantly more likely to occur by jumping and less likely to occur by charcoal burning [19] compared 672 bipolar disorder suicides with suicides from the general population and found that jumping was significant.

The periods during and following hospitalization represent high risk times for suicidal act among bipolar disorder patients with $14 \%$ of bipolar disorder suicides occurring during hospitalization with an additional $26 \%$ occurring within the first few weeks after discharge [15].

\section{Materials and methods}

The current study is a descriptive cross sectional study, which was carried out in the inpatient adult psychiatric wards of Al-Maamoura Mental Health Hospital in Alexandria, Egypt. The present study took place from the beginning of March till the end of August 2018. The number of patients who participated in the research and finished all the psychometrics were 113 patients, 73 males and 40 females, included patients of both sex, aged between 18 t 60 years old, without systemic disease or comorbid mental disorder and patients with clinically detected average intelligence.

All cases were subjected to psychiatric sheet of General Secretariat of Mental Health, Structured interview using Diagnostic and Statistical Manual of Mental Disorders, Fourth Edition (DSM IV) criteria for diagnosis of bipolar disorder and SCID I, General physical and neurological examination, mental state examination and the Arabic version of Beck Scale for Suicide Ideation (BSS) [1] which contains 21 statements each assessing various aspects of suicidal ideation were 
done. The answers were assessed as follows, patients without suicidal risk, with passive ideation, with active ideation and with active and passive suicidal ideation.

Data were fed to the computer and analyzed using IBM SPSS software package version 20.0. [16]. Qualitative data were described using number and percent. Quantitative data were described using range (minimum and maximum), mean, standard deviation and median. Significance of the obtained results was judged at the $5 \%$ level.

\section{Results}

Table (1) Socio-demographic data of bipolar patients studied $(n=113)$.

\begin{tabular}{lcc}
\hline & No & \% \\
\hline Sex & 73 & 64.6 \\
Male & 40 & 35.4 \\
Female & & \\
Age (years) & 47 & 41.6 \\
<30 & 58 & 51.3 \\
30-50 & 8 & 7.1 \\
$>\mathbf{5 0}$ & & \\
Occupation & 24 & 21.2 \\
Clerk jobs & 21 & 18.6 \\
Handicraft & 57 & 50.4 \\
Unemployed & 3 & 2.7 \\
Student & 8 & 7.1 \\
Miscellaneous & & \\
Smoking & 45 & 39.8 \\
Non smoker & 68 & 60.2 \\
Smoker & & \\
Residence & 91 & 80.5 \\
Urban & 22 & 19.5 \\
Rural & & \\
Education & 13 & 11.5 \\
Illiterate & 10 & 8.8 \\
Primary & 24 & 21.2 \\
Preparatory & 53 & 46.9 \\
Secondary & 13 & 11.5 \\
University & & \\
\hline
\end{tabular}

Table (2) Distribution of the studied cases according to Beck Scale for Suicide Ideation (BSS) $(n=113)$.

\begin{tabular}{lcc}
\hline Beck scale & No. & \% \\
\hline No suicidal ideation & 58 & 51.3 \\
Passive ideas & 30 & 26.5 \\
Active ideas & 19 & 16.8 \\
Active and passive ideas & 6 & 5.3 \\
\hline
\end{tabular}

Table (3) Relation between Beck scale for suicide ideation (BSS) and socio-demographic data $(\mathrm{n}=113)$.

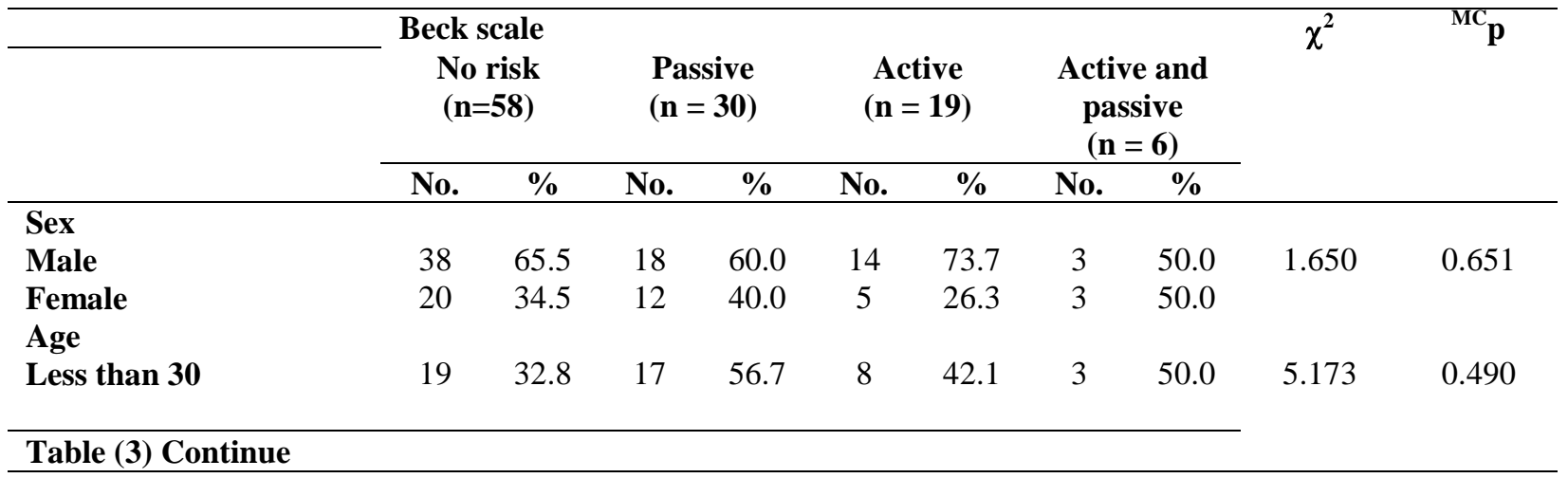




\begin{tabular}{|c|c|c|c|c|c|c|c|c|c|c|}
\hline 30-50 & 34 & 58.6 & 11 & 36.7 & 10 & 52.6 & 3 & 50.0 & & \\
\hline More than 50 & 5 & 8.6 & 2 & 6.7 & 1 & 5.3 & 0 & 0.0 & & \\
\hline \multicolumn{11}{|l|}{ Occupation } \\
\hline Clerk jobs & 14 & 24.1 & 5 & 16.7 & 3 & 15.8 & 2 & 33.3 & 8.942 & 0.688 \\
\hline Handicraft & 10 & 17.2 & 6 & 20.0 & 2 & 10.5 & 3 & 50.0 & & \\
\hline Unemployed & 28 & 48.3 & 16 & 53.3 & 12 & 63.2 & 1 & 16.7 & & \\
\hline Student & 1 & 1.7 & 1 & 3.3 & 1 & 5.3 & 0 & 0.0 & & \\
\hline Miscellaneous & 5 & 8.6 & 2 & 6.7 & 1 & 5.3 & 0 & 0.0 & & \\
\hline \multicolumn{11}{|l|}{ Residence } \\
\hline Urban & 48 & 82.8 & 23 & 76.7 & 15 & 78.9 & 5 & 83.3 & 0.744 & 0.901 \\
\hline Rural & 10 & 17.2 & 7 & 23.3 & 4 & 21.1 & 1 & 16.7 & & \\
\hline Education & & & & & & & & & 9.439 & 0.624 \\
\hline Illiterate & 6 & 10.3 & 3 & 10.0 & 3 & 15.8 & 1 & 16.7 & & \\
\hline Primary & 9 & 15.5 & 0 & 0.0 & 1 & 5.3 & 0 & 0.0 & & \\
\hline Preparatory & 10 & 17.2 & 8 & 26.7 & 4 & 21.1 & 2 & 33.3 & & \\
\hline Secondary & 26 & 44.8 & 16 & 53.3 & 8 & 42.1 & 3 & 50.0 & & \\
\hline University & 7 & 12.1 & 3 & 10.0 & 3 & 15.8 & 0 & 0.0 & & \\
\hline \multicolumn{11}{|l|}{ Onset } \\
\hline Not early onset & 49 & 84.5 & 27 & 90.0 & 14 & 73.7 & 4 & 66.7 & 3.7389 & 0.266 \\
\hline Early onset & 9 & 15.5 & 3 & 10.0 & 5 & 26.3 & 2 & 33.3 & & \\
\hline \multicolumn{11}{|c|}{ Duration (years) } \\
\hline Less than 1 & 9 & 15.5 & 4 & 13.3 & 0 & 0.0 & 1 & 16.7 & & \\
\hline 1 to 10 & 16 & 27.6 & 16 & 53.3 & 12 & 63.2 & 3 & 50.0 & $12.396 *$ & $0.038 *$ \\
\hline More than 10 & 33 & 56.9 & 10 & 33.3 & 7 & 36.8 & 2 & 33.3 & & \\
\hline
\end{tabular}

\footnotetext{
$\chi^{2}$ : Chi square test Mc: Monte Carlo

$p$ : $p$ value for comparing between the different categories

$*$ : Statistically significant at $\mathrm{p} \leq 0.05$
}

\section{Discussion}

However the incidence of bipolar disorder is nearly the same in men and women [6], the present study demonstrates that $64.6 \%$ of the patients were males and $35.4 \%$ were females. The high percentage of males in the current study may be due to the nature of the culture of the society which does not prefer female hospitalization.

Regarding age, it was noted that most of the sample patients were between 30 and $50(51.3 \%)$ and $41.6 \%$ were less than 30 . The least category of the sample was more than 50 (7.1\%) which may be due to complications associated with bipolar disorder which lead to higher mortality rate like accidents and comorbidities, also mental disorders carry a higher risk of death. This came in agreement with [5] who reported that the majority of bipolar patients to be between 19 and 45 .

Regarding residence, it was noted that most of the patients were found to have an urban residence than rural residence which may be due to the geographical location of the hospital from which the sample was taken and also urban areas may be more able to notice the mental abnormalities and seek help rather than rural areas that are inclined to accept the mental disease as a normal process. According to [12] who stated to have a clear and strong signal telling them that urban birth and urban residence associated with a higher risk of developing bipolar disorder.

Regarding employment, it was noted that half of the studied sample $50.4 \%$ were unemployed which could be explained due to the stigma of being a patient and multiple admissions decreasing the employment opportunities, also due to decline of cognitive function with the progress of the disorder and that agrees with [9] who reported that current employment status was significantly related to cognitive functioning, particularly immediate verbal memory. Other factors that affected employment status were severity of symptoms and history of psychiatric hospitalization and their study showed that more than $50 \%$ of bipolar patients were unemployed.

When it comes to the impact on educational level it was noted that $11.5 \%$ reached the university and that was not in agreement with [8] who found that despite comparable IQ levels, patients with bipolar disorder completed fewer years of education than controls. Although more than $60 \%$ of both groups went to college, only $16 \%$ of bipolar patients had a college degree.

Moreover according to beck scale of suicidal ideation 6 patients had active and passive suicidal ideas, 19 patients reported active suicidal ideas, 30patients with passive suicidal ideas and 58 had neither suicidal ideas nor death wishes. This is lower than the numbers reported by [10] who reported that $61 \%$ of bipolar patients had suicidal ideation but the religious background of patients should be put into consideration. Even though bipolar disorder affects the judgment of patients yet the religious ideology of the Egyptian culture still has an impact on most of the patients. 
Unemployed persons have suicidal attempts and ideas more than others, this agree with [18] who reported that unemployment was found to have an association with a two to three folds increased relative risk of death by suicide in comparison to being employed. This may be due to financial difficulties and loss of self-esteem.

Also suicidal ideation are more common in patients with family history of suicide and that agrees with [17] a family history of completed suicide and psychiatric illness was significantly and independently associated with increased risk of suicide.

\section{Conclusions}

From the previous data, the study concluded that the suicidal risk is high among bipolar disorder patients $5.3 \%$ of the studied sample have active and passive suicidal ideas, also unemployment, family history of suicide and longer duration of illness are considered a risk factor for suicidal attempts and ideation .

\section{References}

[1] A.Abdrabboh, S.Elbakry, H.Elsheikh, V.Samy, Suicidal Risk Assessment in Hospitalized Bipolar disorder patients, thesis submitted in Benha university, 2016.

[2] A.R.Singh, towards a suicide free society: Identify suicide prevention as a public health policy, Menssanamonogr, Vol. 2, PP. 21-33, 2004.

[3] B.Kerner, Toward a Deeper Understanding of the Genetics of Bipolar Disorder, Front Psychiatry, Vol. 6, PP. 105, 2015.

[4] B.S.Rudy, Suicide by pedestrian versus motor vehicle: a case report, Am J. Forensic Med Pathol, Vol. 33, PP. 268-69, 2012.

[5] C.D.Mathers, M.Ezzati, Global and regional burden of disease and risk factors: systematic analysis of population health data, Lancet, Vol. 367, PP. 17471757, 2001.

[6] C.K.Farren, K.P.Hill, R.D.Weiss, Bipolar disorder and alcohol use disorder: a review, Current Psychiatry Reports, Vol. 14 (6), PP. 659-66, 2012. [7]D.Osborn, G.Levy, I.Nazareth, M.King, Suicide and severe mental illnesses: Cohort study within the UK general practice research database, Schizophr Res, Vol. 99, PP. 134-138, 2008.
[8] D.C.Glahn, C.E.Bearden, C.L.Bowden, J.C.Soares, Affect Disord. Vol. 92 (2-3), PP. 309-12, 2006.

[9] F.B.Dickerson, J.J.Boronow, C.R.Stallings, A.E.Origoni, S.Cole, R.H.Yoken, Association between cognitive functioning and employment status of persons with bipolar disorder, PsychiatrServ., Vol. 55, PP. 54-58, 2004.

[10] H.Valtonen, K.Suominen, O.Mantere, S. Leppämäki, P.Arvilommi, E.T.Isometsä, Suicidal ideation and attempts in bipolar I and II disorders, 2005.

[11] I.M.Anderson, P.M.Haddad, J.Scott, Bipolar disorder, BMJ (Clinical research ed.), Vol. 345, PP. 8508, 2012.

[12] K.J.T.suchiya, M.Byrne, P.B.Mortensen, Risk factors in relation to an emergence of bipolar disorder: asystematic review, Bipolar Disord, Vol. 5, PP. 231-242, 2003.

[13] M.Pompili, X.Gonda, G.Serafini, Epidemiology of suicide in bipolar disorders: a systematic review of the literature, Bipolar Disord, Vol. 15, PP. 457-490, 2013.

[14] M.A.Ilgen, A.S.Bohnert, R.V.Ignacio, Psychiatric diagnoses and risk of suicide in veterans, Arch Gen Psychiatry, Vol. 67, PP. 1152-1158, 2010.

[15] N.A.Keks, C.Hill, S.Sundram, Evaluation of treatment in 35 cases of bipolar suicid, Australian \& New Zealand J. of Psychiatry, Vol. 43, PP. 503 -508, 2009.

[16] N.Y.Armonk, IBM Corp, Released 2011, IBM SPSS Statistics for windows, vesion 20.0., 2011.

[17] P.L.Qin, E.Agerbo, P.B.Mortensen, Suicide risk in relation to family history of completed suicide and psychiatric disorders: a nested case-control study based on longitudinal registers, 2002.

[18]T.Blakely, S.Collings, J.Atkinson, Unemployment and suicide: Evidence for a causal association, 2003.

[19] U.Ösby, L.Brandt, N.Correia, Excess mortality in bipolar and unipolar disorder in Sweden, Archives of General Psychiatry Vol. 58, PP. 844-850, 2001.

[20] Y.Y. Chen, M.B.Lee, C.M.Chang, S.C.Liao, Methods of suicide in different psychiatric diagnostic groups, J. Affect Disord, Vol. 118(1), PP. 196-200, 2009. 